Das Amidoacettoluid verliert über $150^{\circ}$ erhitzt ein Molekül Wasser und bildet das Hobrecker'sche Aethenyldiamidotoluol, welches durch öftere Sublimation und Krystallisation bei $203^{\circ}$ schmilzt, so wie es Hobrecker angiebt.

Laboratorium der jagiellonischen Universität in Krakau.

273. S. Forsling: Ueber zwei $\beta$-Bromnaphtalinsulfosaluren.

(Eingegangen am 24. Mai; mitgetheilt in der Sitzung von Hrn. W. Will.)

Aus der Brönner'schen $\beta$-Naphtylaminsulfosäure und der Dahl'schen $\beta$-Naphtylaminsulfosäure I habe ich diese beiden $\beta$-Bromnaphtalinsulfosäuren durch die Substitution von Brom für die Amidogruppe erbalten. Auf gewöhnliche Weise, durch die Einwirkung ron rauchender Bromwasserstoffsäure und Kupferbromür auf die Diazoderivate dieser Amidonaphtalinsulfosäuren wurden sie in Bromsulfosäuren übergeführt. Die concentrirten, sauren Lösungen dieser Säuren wurden mit Kaliumcarbonat neutralisirt, wobei die Kaliumsalze ausfielen. Aus diesen Salzen sind die übrigen Derivate der Säuren dargestellt.

I. $\beta_{1}=\beta_{3}$-Bromnaphtalinsulfosänare

Diese Säure wurde aus der Brönner'schen Sulfosäure ${ }^{1}$ ) hergestellt, welche man als ein $\beta_{1}=\beta_{2}$-Derivat annimmt. Die freie Bromsulfosäure ist in Wasser äusserst leicht löslich. Ihre Salze sind dagegen hierin ziemlich schwer löslich. Die Kalium - und Ammonsalze lösen sich am leichtesten, weshalb die übrigen aus diesen Salzen durch Zusatz von leicht löslichen Metallsalzen dargestellt werden können.

Kaliumsalz, $\mathrm{C}_{10} \mathrm{H}_{6} \mathrm{BrSO}_{3} \mathrm{~K}+1 / 2 \mathrm{H}_{2} \mathrm{O}$, bildet ein mikrokrystallinisches Pulver. Ueber Schwefelsäure getrocknet, verlor es beim Erhitzen auf $100^{\circ}$ C. 1/2 Mol. Krystallwasser.

Analyse:

$\begin{array}{lcc} & \text { Gefonden } & \text { Berechnet } \\ \mathrm{K} & 11.62 & 11.70 \text { pCt. } \\ \mathrm{H}_{2} \mathrm{O} & 2.71 & 2.69\end{array}$

Ammonsalz, $\mathrm{C}_{10} \mathrm{H}_{6} \mathrm{BrSO}_{3} \mathrm{NH}_{4}$, ist dem Kaliumsalze ähnlich. Das Salz ist wasserfrei.

1) Diese Berichte XX, 76. 
Analyse:

$\begin{array}{ccc} & \text { Gefunden } & \text { Berechnet } \\ \mathrm{N} & 4.44 & 4.60 \mathrm{pCt} .\end{array}$

Das Baryumsalz wurde aus dem Kaliumsalz mit Chlorbaryum dargestellt. Es fällt als ein mikrokrystallinisches Pulver. Das Silber 8 alz, aus dem Kalinmsalz mit $\mathrm{AgNO}_{3}$, krystallisirt in feinen, weissen, seideglänzenden Nädelchen:

Bromid der $\beta_{1}=\beta_{3}$-Bromnaphtalinsulfosäure.

Das obenerwähnte Kaliumsalz wurde auf gewöhnliche Weise mit Phosphorpentabromid bebandelt. Das Reactionsproduct, welches eine feste gelbe Masse bildete, wurde mit Wasser bebandelt und darauf in Benzol gelöst. $\mathrm{Zu}$ dieser Benzollösung wurde Ligroïn gefügt, wobei die Verunreinigungen ausfielen. Nach dem Concentriren der Benzolligroïnlösung krystallisirte das Bromid in schwach gelben, festen Aggregaten von kleinen Nadeln. Das Bromid löst sich leicht in Benzol und Chloroform; in Ligroïn und in Aether ist es dagegen schwer löslich. Aus einer Mischung von Aether und Chloroform krystallisirte es in Prismen von dem Schmelzpunkt $118^{\circ} \mathrm{C}$.

Analyse :

$\begin{array}{ccc} & \text { Gefunden } & \text { Berechnet } \\ \text { Br } & 45.87 & 45.71 \mathrm{pCt} .\end{array}$

Amid der $\beta_{1}=\beta_{3}$-Bromnaphtalinsulfosäure.

Das Bromid wurde mit einer Mischung von Alkohol und Ammoniak gekocht, worin es sich leicht löste. Nach dem Erkalten krystallisirte das Amid in langen feinen Nädelchen aus. In absolutem Alkohol löst es sich leicht. Es schmolz bei $207^{\circ} \mathrm{C}$.

Analyse:

$$
\begin{array}{ccr} 
& \text { Gefunden } & \text { Berechnet } \\
\mathrm{N} & 5.22 & 4.90 \text { pCt. } \\
& \multicolumn{2}{c}{\beta_{1}=\beta_{3} \text {-Dibromnaphtalin. }}
\end{array}
$$

Das Bromid wurde mit der berechneten Menge Phosphorpentabromid in einer Retorte destillirt. Das so erhaltene Dibromnaphtalin wurde aus Alkohol krystallisirt und darauf mit Wasserdampf destillirt. Das Dibromnaphtalin ist in Alkohol ziemlich schwer löslich. Es löst sich leicht in Ligroïn, Aether und Chloroform. Aus einer Mischung von Aether und Chloroform krystallisirt es in vierseitigen farblosen Tafeln. Schmelzpunkt $158^{\circ} \mathrm{C}$.

Analyse:

$$
\begin{array}{ccc} 
& \text { Gefunden } & \text { Berechnet } \\
\mathrm{Br} & 56.26 & \mathbf{5 5 . 9 4} \mathrm{pCt} .
\end{array}
$$

Das entsprechende Dichlornaphtalin ist $\varepsilon$-Dichlornaphtalin(Schmelzpunkt $\left.136^{\circ}\right)$, welches ich früher aus der Brönner'schen Sulfosäure 
hergestellt habe. Beim Sulfoniren des $\alpha$-Bromnaphtalins mit rauchender Schwefelsäure und Behandeln der so gebildeten Sulfosäuren mit Phosphorpentachlorid hat Jolin ${ }^{1}$ ) neben $\beta$-Dibromnaphtalin (Schmelzpunkt $80.5^{\circ}$ ) ein bei $159.5^{\circ}$ schmelzendes Dibromnaphtalin, $\varepsilon$-Dibromnaphtalin, erhalten. Indessen war das Material, von welchem Jolin ausging, nicht rein. Bei Wiederholung dieser Operation mit reinem Material erhielt er nur $\beta$-Dibromnaphtalin. Jolin nabm dann an, dass das $\alpha$-Bromnaphtalin beim Behandeln mit rauchender Schwefelsäure zwei Sulfosäuren giebt. Weil er aber nur aus dem unreinen Material $\varepsilon$-Dibromnaphtalin erhielt, ist es zu vermuthen, dass das $\alpha$-Bromnaphtalin mit $\beta$-Bromnaphtalin verunreinigt war und die Bromnaphtalinsulfosäure, aus welcher er $\varepsilon$-Dibromnaphtalin erhielt, eine $\beta$-Bromnaphtalinsulfosäure war. Wegen des hohen Schmelzpunktes dieses Dibromnaphtalins und wegen seiner grossen Aehnlichkeit mit dem meinigen sind diese Dibromnaphtaline wahrscheinlich identisch. Ihre Constitution ist $\beta_{1}=\beta_{3}$.

\section{II. $\beta_{1}=\alpha_{4}-$ Bromnaphtalinsulfosäure.}

Diese Bromnaphtalinsulfosäure habe ich aus meiner $\beta$-Naphtylaminsulfosäure $I^{2}$ ), welche mit der Dahl'schen Sulfosäure I ( $\beta$-Naphtylamin- $\alpha$-sulfosäure) identisch ist, hergestellt.

Kaliumsalz, $\mathrm{C}_{10} \mathrm{H}_{6} \mathrm{BrSO}_{3} \mathrm{~K}+\mathrm{H}_{2} \mathrm{O}$, ist dem Kaliumsalz der $\not_{1}=\beta_{2}$-Bromnaphtasulfosäure ähnlich. Ueber Schwefelsäure getrocknet verlor das Salz beim Erhitzen auf $150^{\circ} 1$ Mol. Krystallwasser.

Analyse:

$\begin{array}{lcc} & \text { Gefunden } & \text { Berechnet } \\ \mathrm{K} & 11.38 & 11.42 \mathrm{pCt} . \\ \mathrm{H}_{2} \mathrm{O} & 5.31 & 5.25\end{array}$

Bromid der $\beta_{1}=\alpha_{4}$-Bromnaphtalinsulfosäure.

Auf gewöbnliche Weise dargestellt, wurde das Bromid aus einer Mischung ron Benzol und Ligroïn krystallisirt. Das Bromid löst sich leicht in Benzol und Chloroform. In Ligroïn und in Aether ist dasselbe schwer löslich. Aus Ligrö̈n krystallisirt es in festen Aggregaten, aus Chloroform in breiten Nadeln rom Schmelzpunkte $151^{\circ} \mathrm{C}$.

Analyse:

$$
\begin{array}{ccc} 
& \text { Gefunden } & \text { Berechnet } \\
\mathrm{Br} & 46.00 & 45.71 \mathrm{pCt} .
\end{array}
$$

Dibromnaphtalin.

Das Bromid wurde mit Phosphorpentabromid destillirt. Das so erhaltene Dibromnaphtalin wurde aus Alkohol krystallisirt und mit

1) Nova Acta Soc. Sc. Upsali 1877.

7) Diese Berichte XX, 2099. 
Wasserdampf destillirt. In Alkohol ist es ziemlich schwer löslich. In Aether und Chloroform löst es sich leicht. Es krystallisirt in weissen Nädelchen. Schmp. $75^{\circ} \mathrm{C}$.

Analyse:

$$
\begin{aligned}
& \text { Gefunden Berechnet } \\
& \mathrm{Br} \quad 55.50 \quad 55.94 \mathrm{pCt} \text {. }
\end{aligned}
$$

Dieses Dibromnaphtalin ist früher von Meldola ${ }^{1}$ ) und von Darmstädter and Wichelhaus ${ }^{2}$ ) erhalten. Bei der Oxydation desselben hat Meldola eine Monobromphtalsäure erhalten, welche er als ein 1-,2-, 3-Derivat annimmt. Das entsprechende Dichlornaphtalin ist 8-Dichlornaphtalin (Schmelzpunkt $62^{\circ} \mathrm{C}$.), welches nach Erdmann ein $\beta_{1}=\alpha_{4}$-Derivat ist. Die Bromnaphtalinsulfosäure ist deshalb<smiles>O=S(=O)(O)C1CCCC2CCC(Br)CC21</smiles>

Upsala, im April 1889. Universitätslaboratorium.

274. A. Ladenburg: Nachtrag zu der Mittheilung uiber die Synthese der activen Coniine.

(Eingegangen am 29. Mai.)

Als ich vor einigen Jahren die Synthese des Coniins entdeckt hatte, lag mir viel daran zu zeigen, dass damit die erste rollständige Synthese eines Alkaloïds gelungen war. Ich habe deshalb in dem Vortrag, den ich damals auf der Naturforscherversammlung in Berlin hielt, gerade diesen Punkt besonders betont und ausgeführt, und in dem Referat, welches die Chemiker-Zeitung von diesem Vortrag brachte (3. October 1886), findet sich auch der Nachweis, dass diese Synthese wirklich eine vollständige gewesen ist.

Als ich später in diesen Berichten meine Untersuchung mittheilte, habe ich diese Synthese als eine vollständige bezeichnet, aber nicht geglaubt, dies durch Belege erweisen zu müssen, und ebenso wenig habe ich dies in den Annalen der Chemie gethan, in welchen ich meine Untersuchungen über Pyridin- und Piperidinbasen im Zusammenhang dargestellt habe.

1) Journ. of the Chem. Soc. 1885, 497.

2) Ann. Chem. Pharm. 152, 298. 